\title{
DEVELOPMENT AND CHARACTERIZATION OF STITCH-BASED SENSORS
}

\section{DOI: 10.35530/TT.2021.08}

\section{S.A. Odhiambo*, S. Vasile, J. Sarrazyn, I. Rottiers, A. De Raeve}

FTI Lab ${ }^{+}$, HOGENT University of Applied Sciences \& Arts, Belgium

(E-mail: sheilla.odhiambo@hogent.be, simona.vasile@hogent.be, ingeborg.rottiers@hogent.be, jochen.sarrazyn@hogent.be, alexandra.deraeve@hogent.be)

\begin{abstract}
Strain sensing seams have been developed by integrating conductive sewing threads in different types of seam designs on a fabric typical for sports clothing using sewing technology. The aim was to obtain a simply integrated stitch-based sensor that can be applied on sports clothing to monitor the movements of the upper body parts of the user during exercising. Stitch types 304; 406; 602 and 605 were produced. The seams were made on a knitted fabric composed of $80 \%$ polyamide 6.6 and $20 \%$ elastane. The seams underwent stretch cycling for 10 cycles and up to 44 cycles following EN ISO 14704-1:2005 (modified), using an INSTRON tensile tester machine. The changes in the resistance of the seams with time were recorded simultaneously using Agilent meter U1273A. Sensing functionality among which is sensor gauge factor (GF), stability, drift, and reproducibility were evaluated on the promising sensor seams. The type of base fabric used, stitch type, stitch formation process (friction and dynamic forces during sewing), integrated EC thread length, and positioning of thread(s) in the fabric have a significant influence on the performance of the seams. Sensor seam 406001comprising 2 EC yarns (Madeira HC12) and Sensor seam 304-010 comprising 1 EC yarn (Madeira HC40) turned out to be very promising and others shall be improved (sensor 602-006 with Madeira HC 40 and sensor 605-002 with a Muriel yarn).
\end{abstract}

Keywords: electro-conductive sewing thread, sensing seams, sewing technology, stitch structure, electrical resistance

\section{INTRODUCTION}

The recent rise of personalized wearable devices that can monitor body parameters while sporting has made it increasingly important to have flexible textile-based sensor alternatives that can be comfortably worn [1-3]. Textile strain sensors offer a new generation of devices that combine strain sensing functionality with wearability and high stretchability [4-7]. Previously, sensors have been integrated into the textile structure before garment production, through printing [5], coating [8-10], weaving [11], or knitting [4]. These high levels of sensor integration mostly face challenges related to sensor connection with other parts of the smart systems [12] and also make potential garment repairing or disassembling for recycling purposes at the end of life difficult.

Stitch-based strain sensors are easily embedded in the garment and connected with other parts of the smart system such as the processing and communicating unit. Moreover, their integration into the garments towards the end of the production process offers definite advantages in terms of flexibility and production costs. Tangsirinaruenart \& Stylios [13] employed sewing technology to develop strain sensors based on two specific 
conductive sewing threads and several stitch types. Also, Gioberto et al. [14] confirm that most common sewing machines (the cover stitch and overlock machines) can produce reliable, repeatable, sensitive sensors. Each sewing machine produces sensors with slightly different characteristics and parameters. The tension of the conductive yarn during lockstitching is an important factor to control the sensitivity of the strain sensor [15].

Despite the potential of this technology, the number of studies investigating stitchbased sensors is rather limited. Therefore this research aims to investigate some commercially available electro-conductive (EC) sewing threads, assess their properties, sewability and characterize the performance of their various stitch-based sensor configurations.

\section{MATERIALS AND METHODS}

\subsection{Electro-conductive (EC) sewing threads and their characterization}

Commercial EC threads with various compositions were selected and their properties were assessed: linear density (ISO 2060: 1994), linear electrical resistance (EN 16812:2016), tensile strain, and load at break (ISO 2062: 2009). A knitted fabric typically used in sports clothing, (80/20 PA/ EL) was used as the substrate for the strain sensor seams. The fabric was characterized to determine its weight per unit area (ISO 3801: 1977), thickness (ISO 5084: 1996), and elasticity (EN ISO 14704-1: 2005). We informally evaluated the suitability of sewing the selected EC yarns on conventional sewing machines and used in some cases silicone oil to smoothen the sewing process.

\subsection{Seams and stitch-based sensors characterization}

In the production of the sensing seams, EC threads were mostly used as bobbin threads aiming at low friction and low thread tension, in some cases the EC thread could be used as a needle thread too. Stitch types 304, 406, 602, and 605 were produced and the successfully stitched sensors were characterized using an experimental set-up similar to [13], using a tensile tester Instron and an Agilent meter U1273A. Rectangular fabric strips of $5 \times 20 \mathrm{~cm}$ containing the sensor seam were fixed between the machine clamps at a gauge of $100 \mathrm{~mm}$. The resistance response of the sensors according to the applied strain force was recorded upon several stretch cycles performed to a maximum load of $35 \mathrm{~N}$ with a speed of $500 \mathrm{~mm} / \mathrm{min}$ (EN ISO 14704-1: 2005). Several specimens were tested to assess potential variations within the same type of sensor configuration and to assess the reproducibility of the sensors. The sensors underwent 10 cycles to assess their sensitivity and 44 cycles to assess their stability i.e. 44 cycles is the maximum number of cycles for the tensile tester Instron machine. The average time per cycle (loading and offloading)was 12 seconds. The initial sensor seam electrical resistance (R0) was noted for each sensor just before the onset of cycling. The changes in resistance and strain were recorded continuously throughout the stretch cycling. The sensor gauge factor (GF) was determined at the $2^{\text {nd }}, 10^{\text {th, }}$ and $44^{\text {th }}$ cycles. The GF represents the sensitivity of the sensor and was calculated as the ratio of changes in resistance at any given cycle relative to R0, to the relative strains. The drift of the GF between the $2^{\text {nd }}$ and $44^{\text {th }}$ cycles was also determined. These parameters indicate the sensor's sensitivity, stability, and reproducibility.

\section{RESULTS AND DISCUSSIONS}

The linear density (count) of the EC threads ranged between 260-2000 dtex, their resistance between $82-2000 \mathrm{ohm} / \mathrm{m}$, and they had an elongation (strain at break) between 
18 - $36 \%$ (table 1). The knitted fabric (substrate) had a mass per unit area of $276 \pm 5 \mathrm{~g} / \mathrm{m}^{2}$, a thickness of $0.46 \mathrm{~mm}$, and exhibited a tensile strain of $132.4 \pm 3.1 \%$ and $150 \pm 6.6 \%$ in the wales and course direction respectively.

In some cases, the sewability of EC threads was challenging, adjustments of the machine speed and use of silicon oil were necessary to avoid irregular stitches. The density of the base fabric affected the precision of the stitches, doubling the fabric in some cases enabled more accurate and neat stitches but affected the stretchability of the seam. For uniformity purposes, all characterized seams presented hereafter were produced on a single fabric without a backing.

The descriptions of the sensors seams produced are shown in figure 1, the type of the respective EC yarn and the conventional yarns used in each seam, the stitch dimensions, and the images of the front and the back of the seams. Visual inspection of the seams after cycling shows that the main fabric is elongating as well as narrowing during cycling. This means that any portion of the EC thread in the direction of the stretch is also elongated.

Table 1. Properties of selected EC threads

\begin{tabular}{|c|c|c|c|c|}
\hline EC thread reference (company) & EC thread composition & $\begin{array}{l}\text { Count } \\
\text { (dtex) }\end{array}$ & $\begin{array}{c}\text { Resistance } \\
(\Omega / \mathbf{m})\end{array}$ & $\begin{array}{c}\text { Strain at break } \\
(\%)\end{array}$ \\
\hline Silvertech 120 (Amann) & $\begin{array}{l}\text { Silver coated PA/PES } \\
\text { hybrid thread (spun) }\end{array}$ & 260 & 530 & 17 \\
\hline Madeira HC 40 & Silver plated PA (spun) & 300 & $<300 *$ & 20 \\
\hline Madeira HC 12 & Silver plated PA (spun) & 630 & $<100^{*}$ & 25 \\
\hline $\begin{array}{c}\text { Soieries Elite Elinox } \\
\text { 3x(PESHT140dtex2VN35) }\end{array}$ & $\begin{array}{l}\text { (Twisted PES/ } 2 \text { SS } \\
\text { filaments) x } 3 \text { doubled }\end{array}$ & 900 & 134 & 14 \\
\hline $\begin{array}{c}\text { Soieries Elite Elinox } \\
\text { 5x(PESHT140dtex2VN35) }\end{array}$ & $\begin{array}{l}\text { (Twisted PES/2 SS } \\
\text { filaments) x } 5 \text { doubled }\end{array}$ & 1980 & 82 & 15 \\
\hline $\begin{array}{c}\text { Soieries Elite Elinox } \\
\text { PESHT140dtex2VN35 }\end{array}$ & $\begin{array}{c}\text { Twisted PES/ SS } \\
\text { filaments }\end{array}$ & 1780 & 100 & 16 \\
\hline $\begin{array}{c}\text { Soieries Elite Elinox } \\
\text { PESHT280dtexVN35PES20dtex }\end{array}$ & $\begin{array}{l}\text { Twisted PES/ SS } \\
\text { filaments }\end{array}$ & 430 & 865 & 17 \\
\hline Lemur Muriel sensor yarn 70Sh & $\begin{array}{c}\text { Elastic conductive } \\
\text { silicone monofilament }\end{array}$ & $2000 *$ & $2000^{*}$ & $300 *$ \\
\hline
\end{tabular}

*Asterisk shows manufacturers specifications: PES - polyester; PA - Polyamide; SS - filaments stainless steel VN35 (Bekintex)

The strain sensor performance depended on the resistance per meter (ohm/meter) of the EC yarn(s) used/employed (mainly 1 EC yarn), the length of the EC yarn in the seam length of $100 \mathrm{~mm}$, the number of contact points within the seam length, the seam stretchability, and the base fabric stretchability. The initial resistance of the seam just before loading depended on the total yarn length in the seam concerning the effective current path. Thus complexly configured seams with many flexible loops tend to comprise more EC yarn length with more flexible contact points thus unpredictable behavior of the strain sensor. The variation in sensitivity could also be due to irregularities in the stitches during production or the EC yarn imperfection at microscopic levels.

The gauge factor (GF) is the most important performance parameter which is a dimensionless value that determines the sensitivity of a sensor. The gauge factor of the strain sensors at the $2^{\text {nd }}, 10^{\text {th, }}$ and $44^{\text {th }}$ cycles was calculated by equation 1 :

$$
G F=\frac{\Delta \mathrm{R} / \mathrm{RO}}{\varepsilon}=\frac{\Delta \mathrm{R} / \mathrm{R} 0}{\Delta \mathrm{L} / \mathrm{LO}}
$$


where R0 and L0 are the unstretched resistances and length respectively; $\Delta \mathrm{R}$ and $\Delta \mathrm{L}$ are the changes in resistances and length due to stretching respectively; $\boldsymbol{\varepsilon}$ is the strain. Higher initial resistance means that for a given gauge factor changes in strain would produce larger changes in resistance. However high resistance sensors also tend to have a much lower gauge factor so offsetting this advantage.

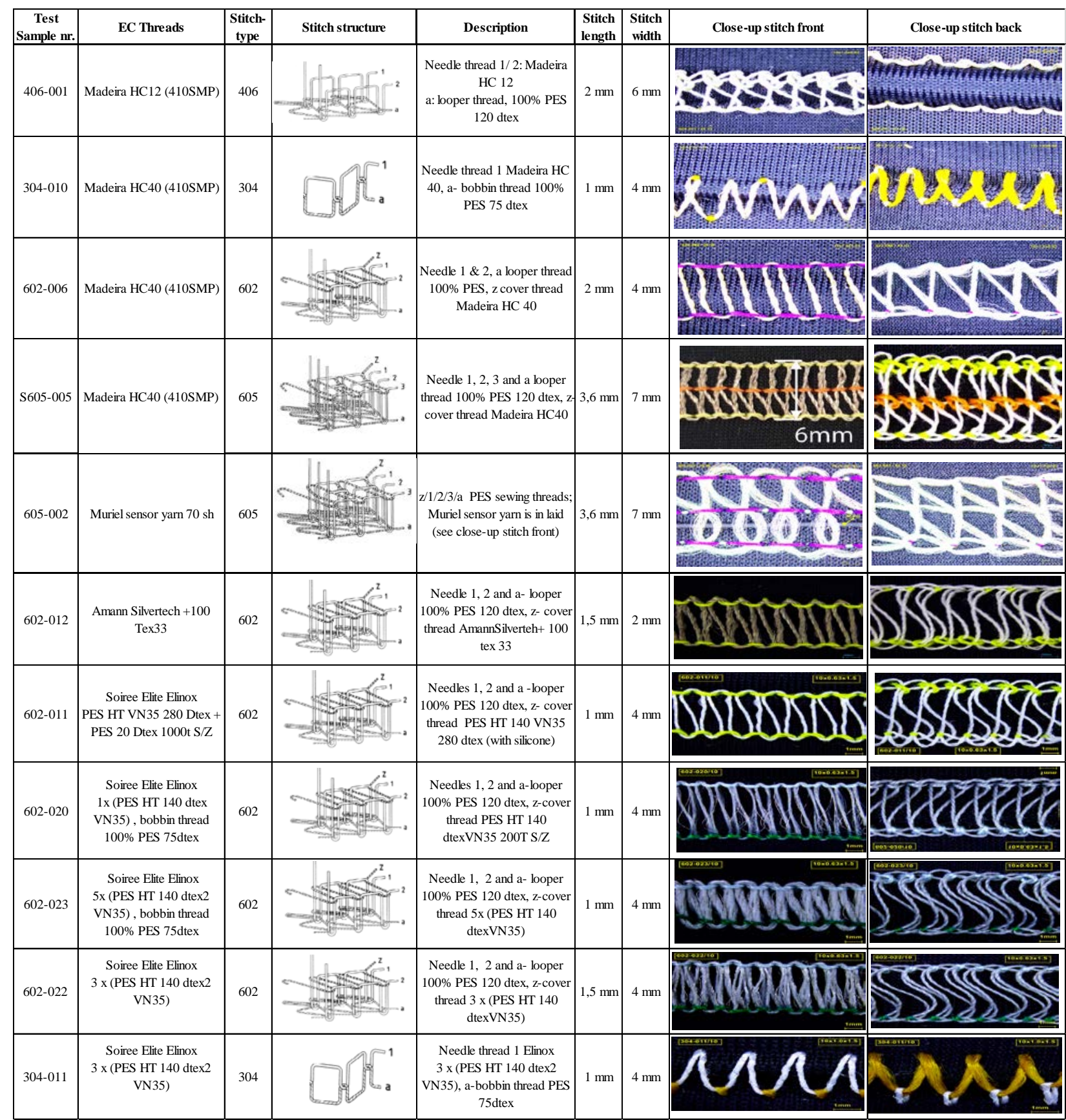

Figure 1. Configurations of the produced sensor seams

Linearity between seam resistance and absolute strain during loading and offloading in the cycling process, repeatability of the loading-offloading patterns for the consequent cycles (up to 44), and reproducibility of the strain sensor seams were key aspects in selecting the performing sensors. The selected sensors presented in this paper exhibited 
these properties, however, each sensor could hardly return to its exact previous state during cycling, hence slight drifts were observed in the consecutive cycles. The drift was calculated as the differences in the change in resistance at the peak of the second cycle and the peaks of the respective cycles (the $10^{\text {th, }}$ or the $44^{\text {th }}$ cycles). The summary of the GF values and Drifts at the respective cycles is shown in table 2.

Not all seams produced real strain sensors, some seams could barely show any response during stretch cycling. Seams with a low response (i.e. low changes of resistance upon stretching) were discarded. Therefore after screening only the most promising sensors have been selected for presentation.

Table 2. Summary of G.F values and drifts of the presented sensors

\begin{tabular}{|c|c|c|c|c|}
\hline EC yarn & Madeira HC 40 & Madeira HC 12 & Muriel sensor yarn 70sh & Madeira HC 40 \\
\hline Stitch number & $304-010$ & $406-001$ & $605-002$ & $602-006$ \\
\hline R0 (Ohm) & 245.38 & 250.5 & 607.92 & 159.63 \\
\hline L0 (nm) & 100 & 100 & 100 & - \\
\hline Strain (\%) cycle 2 & 116.33 & 72 & 84.67 & 63.8 \\
\hline Strain (\%) cycle 10 & 121 & 74 & 101.92 & - \\
\hline Strain (\%) cycle 44 & 124 & 75 & - & 0.53 \\
\hline$\Delta$ R2/R2 (\%) & 11.1 & 32.47 & 149.77 & 1.41 \\
\hline$\Delta$ R10/R0 (\%) & 11.5 & 38.46 & 197.75 & 0.41 \\
\hline$\Delta$ R44/R0 (\%) & 17.87 & 50.07 & - & 1.08 \\
\hline GF cycle 2 & cycle 2 & 0.45 & 1.77 & - \\
\hline GF cycle 10 & 0.10 & 0.52 & - & 0.6769 \\
\hline GF cycle 44 & 0.14 & 0.67 & 0.1714 & - \\
\hline GF drift cycle 10 & -0.0004 & 0.0688 & - & 67.69 \\
\hline GF drift cycle 44 & 0.049 & 0.217 & 17.14 & - \\
\hline GF drift (\%) cycle 10 & -0.04 & 6.88 & - & \\
\hline GF drift (\%) cycle 44 & 4.90 & 21.66 & & \\
\hline
\end{tabular}

The sensor performance varied depending on stitch type to EC-thread combination. For instance, sensor seam 406-001 (comprising two EC threads Madeira HC12) had a good performance. This sensor seam showed linearity during cycling (the proportional changes in resistances during loading and offloading to the changes in the strain) and stability in consequent cycles (the same cycle pattern was observed throughout the cycling). Three different samples of the same were compared and the results showed consistency. The sensor seam is reproducible and showed low variation among the three specimens, its resistance varied relatively narrowly between 300-350 ohm (figure 2, a). This seam reached an elongation of $80 \%$ during stretch cycling (figure 2, b) at a maximum load of $35 \mathrm{~N}$ and its GF was 0.52 at the $10^{\text {th }}$ cycle and 0.67 at the $44^{\text {th }}$ cycle, with a drift of $21 \%$ at the $44^{\text {th }}$ cycle.

Sensor Seam 304-010 (comprising one EC yarn Madeira HC40) was very elastic with maximum elongation of $120 \%$, at $35 \mathrm{~N}$ (figure 3, b), and could withstand 44 cycles with only $4.8 \%$ drift in GF at the $44^{\text {th }}$ cycle. A sample of this sensor seam was very stable and had a very low variation of the sensing response between cycles 1-44. However, its GF was low (about 0.1). Figure 3, a shows that its resistance varied narrowly (250-270 $\mathrm{Ohm}$ ) and that it exhibited low variation between the three similar specimens tested hence shows good reproducibility. Figure 3, b shows that this sensor is stable up to 44 cycles. 


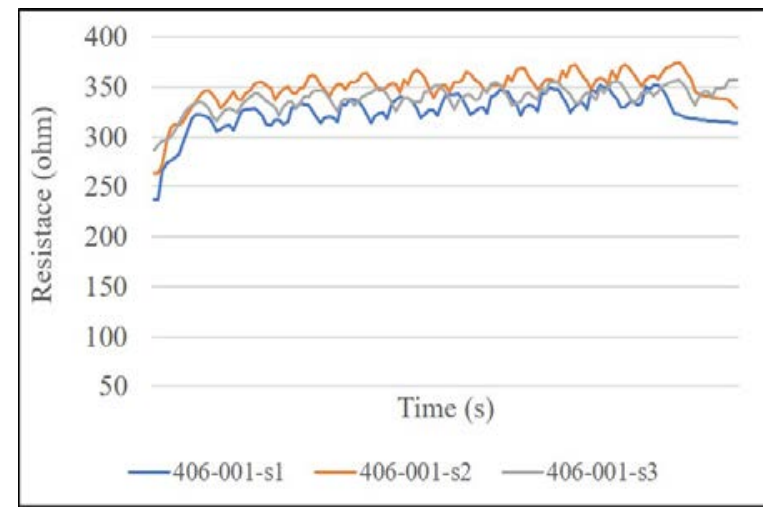

a

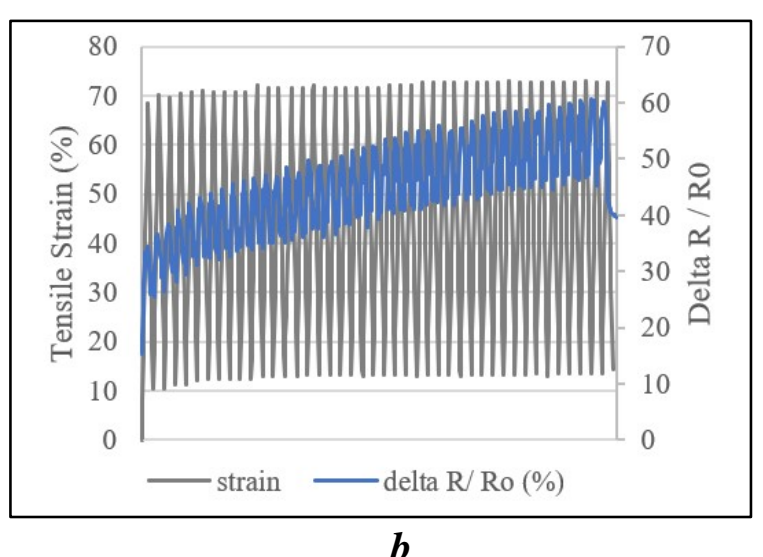

Figure 2. Seam 406-001 performance for the three specimens: a - comparison of s1, s2, and s3 changes in electrical resistance in time of cycling among samples ( reproducibility);

B - sensor seam stability up to 44 cycles

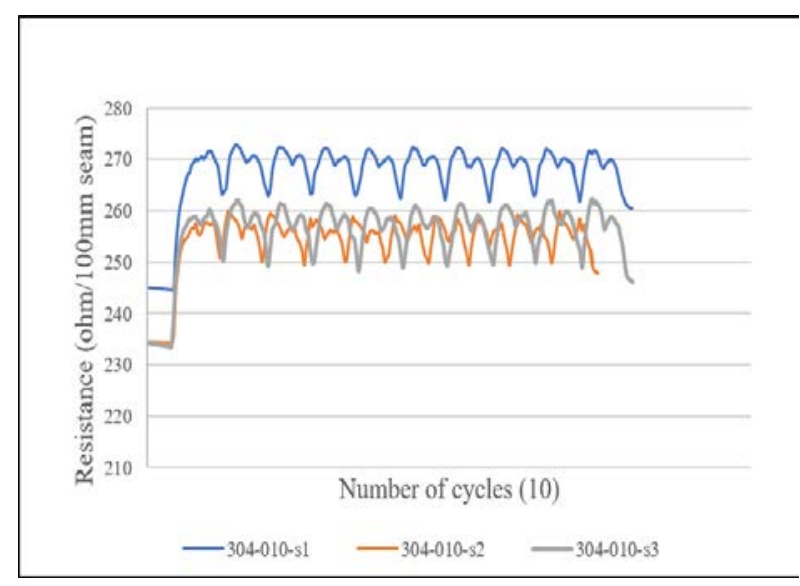

a

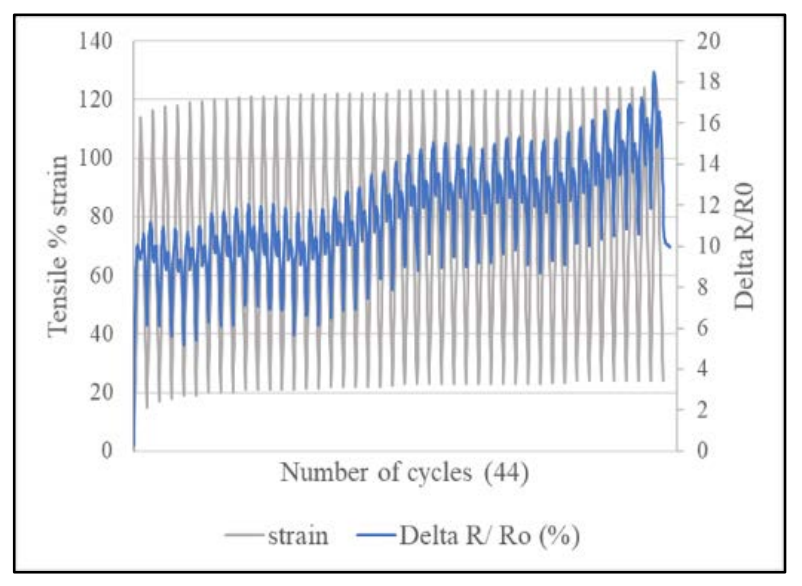

b

Figure 3. Seam 304-010 performance for the three specimens: a - comparison of s1, s2, and s3 changes in electrical resistance in time of cycling among samples ( reproducibility); b - sensor seam stability up to 44 cycles

Sensor seam 602-006 (comprising one EC-yarn Madeira HC 40) was less elastic (around $60 \%$ at $35 \mathrm{~N}$ ) and its resistance varied very narrowly (160-170 ohm) within the 10 cycles as shown in figure 4 . The sensor GF was 0.41 at the $2^{\text {nd }}$ cycle and 1.08 at the $10^{\text {th }}$ cycle) with a high drift of $68 \%$, at the $10^{\text {th }}$ cycle (it can be seen in figure 4 , b that the peak of the strain or delta R/R0 shifts with an increase in the number of cycles). However, this sensor had good reproducibility (figure 4 , a).

Sensor seam 605-002 (comprising one Muriel yarn, laid in the seam, on the fabric surface) exhibited very high sensitivity. Controlling the yarn tension during sewing was very difficult and this resulted in large variation among identical samples as can be seen in figure 5, a. Nevertheless, the sensitivity pattern of the first 10 cycles was similar for both samples with large strains. These seam sensors could easily stretch up to $100 \%$ of the original length at the given load of $35 \mathrm{~N}$ and exhibited very high variation in delta R/R0 after 10 cycles. The sensor GF was1.77 at the 2nd cycle and 1.94 at the 10 th cycle with a high drift of $17 \%$, at the 10 th cycle. 


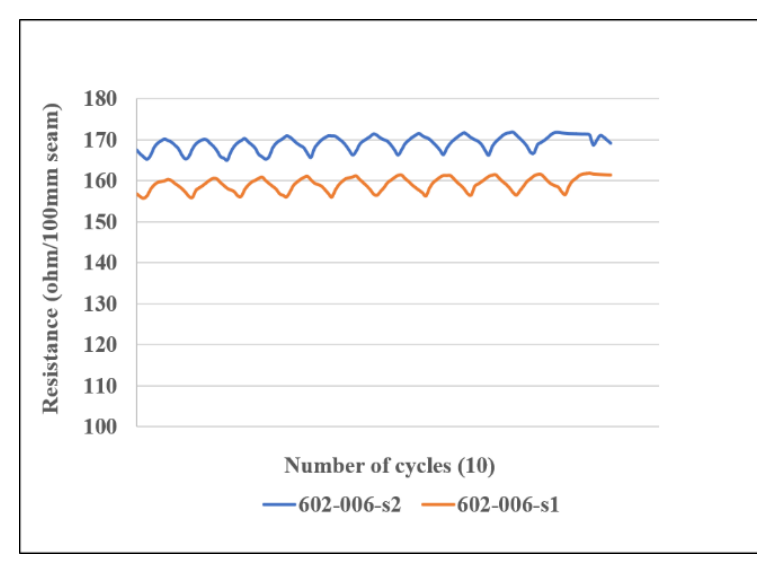

a

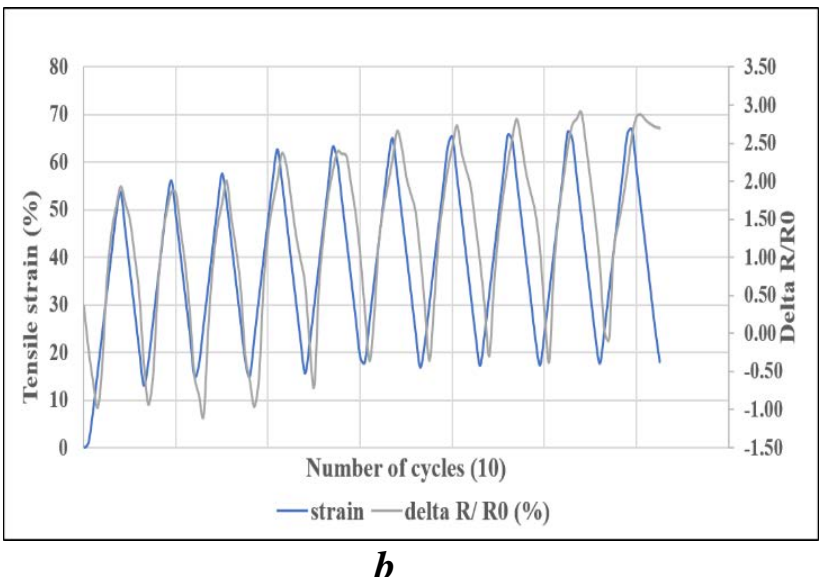

b

Figure 4. Seam 602-006 performance for the two specimens: a - comparison of s1 and s2 changes in electrical resistance in time of cycling among samples ( reproducibility); b - drift in the gauge factor with the increase in the number of cycles within sample 2

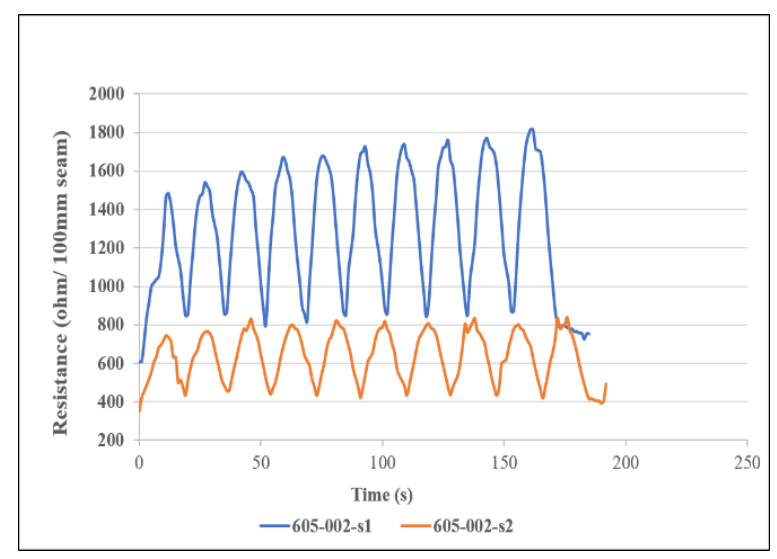

(a)

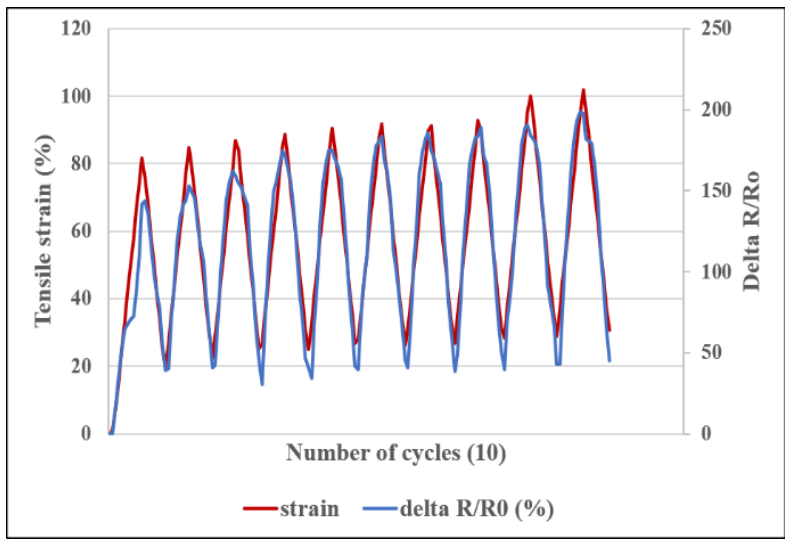

(b)

Figure 5. Seam 605-002 performance for the two specimens; a - comparison of s1 and s2 changes in electrical resistance in time of cycling among samples (reproducibility); b - drift in the gauge factor with the increase in the number of cycles within sample 2

The sensing mechanism is as a result of the alteration of the electromechanical properties of the seams under stress/strain deformation. Therefore the type of base fabric used, stitch type, stitch formation process (friction and dynamic forces during sewing), integrated EC thread length, and positioning of thread(s) in the fabric have significant influence on the performance of the seams during cycling hence an influence on the sensitivity stability and reproducibility of the strain sensor seams.

\section{CONCLUSION}

Different types of EC threads were used to produce various seam types on a fabric substrate typically used for sportswear. The EC yarns were used mostly as bobbin threads to sew them with as little tension and friction as possible.

Sensor seam 406-001comprising 2 EC yarns (Madeira HC12) and Sensor seam 304-010 comprising 1 EC yarn (Madeira HC40) turned out to be very promising and others shall be improved (sensor 602-006 with Madeira HC 40 and sensor 605-002 with a Muriel yarn). Such strain sensors can be potentially used for monitoring body movement 
in sportswear. Further research is needed to optimize configuration EC yarns-fabric-stitch for monitoring movements with the desired stretch, to integrate the sensor, to foresee appropriate connections, and actual assessment of sensor performance and reliability in time and upon garment washing among others.

\section{ACKNOWLEDGMENT}

The authors would like to express appreciation for the financial support of the sponsors VLAIO TETRA programme (B) \& AiF (German federation of Industrial research association) [Project nr. HBC.2019.2679 (478) (CORNET)].

\section{REFERENCES}

[1] Cherenack, K., Pieterson, L.V., Smart textiles: Challenges and opportunities, In: Journal of Applied Physics, 2012, 112, https://doi.org/10.1063/1.4742728

[2] Dervojeda, K., Smart Textiles for Sports; Report on promising KETs-based products, 2021, Available at: https://ec.europa.eu/growth/tools-databases/ketstools/sites/default/files/documents/analytical_report_nr1_smart_textiles_for_sports_final.pdf. [Accessed on June 2021]

[3] Research, T.M., Smart Textile Market - Global Industry Analysis, Size, Share, Growth, Trends and Forecast 2015 - 2023: TMR, 2016, 9/29/2016

[4] Zhang, Q., et al., Textile-Only Capacitive Sensors for Facile Fabric Integration without Compromise of Wearability, In: Advanced Materials Technologies, 2019, 4, 10

[5] Agcayazi, T., et al., Fully-Textile Seam-Line Sensors for Facile Textile Integration and Tunable Multi-Modal Sensing of Pressure, Humidity, and Wetness, In: Advanced Materials Technologies, 2020, 5, 8

[6] Islam, G.M.N., Ali, A., Collie, S., Textile sensors for wearable applications: $a$ comprehensive review, In: Cellulose, 2020, 27, 11, 6103-6131

[7] Guo, L., et al., Improvement of electro-mechanical properties of strain sensors made of elastic-conductive hybrid yarns, In: Textile Research Journal, 2012, 82, 19, 1937-1947

[8] Takamatsu, S., et al., Fabric pressure sensor array fabricated with die-coating and weaving techniques, In: Sensors and Actuators a-Physical, 2012, 184, 57-63

[9] Chatterjee, K., et al., Electrically Conductive Coatings for Fiber-Based E-Textiles, In: Fibers, 2019, 7, 6

[10] Chedid M., Belov, I., Leisner, P., Experimental analysis and modelling of textile transmission line for wearable applications, In: International Journal of Clothing Science and Technology, 2007, 19, 1-2, 59-71

[11] Vasile, S. et al., Study of the contact resistance of interlaced stainless steel yarns embedded in hybrid woven fabrics, In: Autex Research Journal, 2017, 17, 2, 170-176

[12] Matsouka, D., et al., Electrical connection issues on wearable electronics, In: IOP Conference Series-Materials Science and Engineering, 2018

[13] Tangsirinaruenart, O., Stylios, G., A Novel textile stitch-based strain sensor for wearable end users, In: Materials, 2019, 12, 9, 1469

[14] Gioberto, G., et al., Machine-Stitched E-textile Stretch Sensors, In: Sensors \& Transducers, 2016, 202, 7, 25-37

[15] Park, J., et al., Wearable Strain Sensor Using Conductive Yarn Sewed on Clothing for Human Respiratory Monitoring, In: IEEE Sensors Journal, 2020, 20, 21 